(c) 2019. This manuscript version is made available under the CC-BY-NC-ND 4.0 license http://creativecommons.org/licenses/by-nc$\mathrm{nd} / 4.0 /$

\title{
Drained pore modulus and Biot coefficient from pore-scale digital rock simulations
}

\author{
Shakil Ahmed ${ }^{1 \star}$, Tobias M. Müller ${ }^{1 \dagger}$, Mahyar Madadi $^{2 \ddagger}$, Victor Calo ${ }^{3,4,5 \S}$ \\ ${ }^{1}$ Energy, Commonwealth Scientific and Industrial Research Organisation (CSIRO), \\ Kensington, WA 6152, Australia \\ ${ }^{2}$ Exploration Geophysics, Western Australian School of Mines, Faculty of Science and Engineering, \\ Curtin University, Perth, WA 6845, Australia \\ ${ }^{3}$ Applied Geology, Western Australian School of Mines, Faculty of Science and Engineering, \\ Curtin University, Perth, WA 6845, Australia \\ ${ }^{4}$ Mineral Resources, Commonwealth Scientific and Industrial Research Organisation (CSIRO), \\ Kensington, WA 6152, Australia \\ ${ }^{5}$ Curtin Institute for Computation, Curtin University, Perth, WA 6845, Australia
}

1 February 2018

\footnotetext{
* E-mail: Shakil.Ahmed@csiro.au

$\dagger$ E-mail: Tobias.Mueller@csiro.au

‡ E-mail: Mahyar.Madadi@curtin.edu.au

$\S$ E-mail: Victor.Calo@Curtin.edu.au
} 


\section{Ahmed et al.}

\section{Abstract}

Geomechanics helps us understand the life-cycle of a hydrocarbon reservoir and, in turn, impacts geophysical monitoring programs. A common geomechanical problem is to predict reservoir compaction or zones of abnormal pore pressure. These predictions mostly use simple empirical relations, but recently, the use of rock deformation models based on static poroelasticity is the norm. These models require accurate values for the poroelastic parameters. We present a digital rock workflow to determine these poroelastic parameters which are difficult to extract from well-log or laboratory measurements. The drained pore modulus is determinant in the compaction problem. This modulus represents the ratio of pore volume change to confining pressure when the fluid pressure is constant. In laboratory experiments, bulk volume changes are accurately measured by sensors attached to the outer surface of the rock sample. In contrast, pore volume changes are notoriously difficult to measure because these changes need to quantify the pore boundary deformation. Hence, accurate measures of the drained pore modulus are challenging. We simulate static deformation experiments at the pore-scale utilizing digital rock images. We model an Ottawa F-42 sand pack obtained from X-ray micro-tomographic images. We stack two-dimensional micro-CT images to represent a three-dimensional F-42 sand pack sample. We extract a sub-volume from this sample for numerical simulation. We first segment the cropped sample, which consists of grains and pore spaces, and then use the segmentation to generate a volumetric mesh. We compute the elastic, linear momentum balance in the structural domain (grains) and solve the system using the commercial software package ABAQUS. The network of grains (solid phase) is assumed elastic, isotropic, and homogeneous. We calculate the change in pore volume using a new post-processing algorithm, which allows us to compute the local changes in pore volume due to the applied load. This process yields an accurate drained pore modulus. We then use an alternative estimate of the drained pore modulus. We exploit its relation to the drained bulk modulus and the solid phase bulk modulus (i.e., Biots coefficient) using the digital rock workflow. Finally, we compare the drained pore modulus values obtained from these two independent analyses and find reasonable agreement.

Key words: Drained pore modulus, Biot coefficient, digital rocks, poroelasticity. 


\section{INTRODUCTION}

A standard model for the effective elastic behaviour of porous, fluid-saturated rocks is the theory of linear poroelasticity. As it is a macroscopic theory, only an average of the textural and compositional complexities at pore-scale enter the poroelastic description. Since the introduction of the poroelasticity theory by Biot (1941), the definition of the deformation parameters for the constitutive relations has been sought (Biot and Willis, 1957; Makhnenko and Labuz, 2016; and Cheng, 2016 for an overview). We use volume averaging of the pore-scale equations to quantify the parameters appearing in the constitutive equations of linear poroelasticity that include the effects of pore-scale heterogeneities (Sahay et al., 2001; Sahay, 2013; Müller and Sahay, 2013; Müller and Sahay, 2016a).

A poroelastic parameters particularly important for geomechanical reservoir analysis is the Biot coefficient. It is used to estimate the reservoir stress path (Altmann et al., 2010). The Biot coefficient represents the fluid volume change induced by the bulk volume change in drained conditions, which is difficult to measure in the laboratory. An alternative definition of the coefficient is as the effective pressure coefficient for the bulk that is measured in constant bulk volume tests (e.g. Omdal et al., 2009). However, this interpretation is not correct in micro-inhomogeneous rocks (Müller and Sahay, 2016b). Another relevant parameter is the drained pore modulus used in geotechnical applications such as reserve estimation (e.g. Schutjens and Heidug, 2012; De Oliveira et al., 2016). The drained modulus measures the change of pore volume as the confining pressure increases while the fluid pressure is constant. This drained coefficient is as difficult to measure in the laboratory as the Biot coefficient (Pimienta et al., 2017).

Given these problems, we explore new avenues to estimate the Biot coefficient and the drained pore modulus. For idealized, two-dimensional porous structures Tan and Konieztky (2014) compute the Biot coefficient numerically and emphasize that microstructural properties such as the arrangement of elliptical cracks are important. Therefore, we aim at an approach that handles the complexities of a three-dimensional network of grains. In this paper we numerically determine the drained poroelastic parameters using digital rock technology (DRT). DRT technology uses 3D pore-scale images of rocks (i.e., the geometry of the pore-scale features is known and only limited 


\section{$4 \quad$ Ahmed et al.}

by the resolution of the imaging process) in conjunction with numerical solvers for the pore-scale governing equations. However, as Couples (2017) points out, attempts to quantify poromechanical parameters using DRT have not been convincing and remain a major challenge.

The outline is as follows. First, we present the poroelastic framework with particular focus on the interrelations between parameters characterizing the drained rock behaviour. Then, we numerically simulate static deformation experiments at the pore-scale utilizing digitized rock images of sandstone. From the pore-scale simulations we infer the macroscopic deformation moduli by volume-averaging. We interpret these moduli in the poroelastic framework which accounts for micro-inhomogeneities in a generic way and which explicitly links the change of porosity to the constitutive equations of poroelasticity (Müller and Sahay, 2016a). Finally, we compare the numerical estimates with laboratory measurements reported in the literature and obtain a good agreement with these measurements.

\section{MODEL FOR MICRO-INHOMOGENEOUS ROCKS}

\subsection{Constitutive equations of linear poroelasticity and the porosity perturbation equation}

We consider an isotropic poroelastic solid and restrict the analysis to volumetric changes only. This implies the following kinematic variables. The bulk volumetric strain $(\epsilon)$ and the increment of fluid content $(\zeta)$ according to Biot's (1941) consolidation theory, where we adopt the notation of Wang (2000). In addition, since pore boundaries move during deformation (Figure 1) the proportion of solid to fluid volume changes and amounts to a change of porosity $\eta-\eta_{0}$ (Sahay et al., 2001). Here $\eta$ is the porosity at the current state of deformation and $\eta_{0}$ is the porosity at the reference (undeformed) state. While the change of porosity does not appear explicitly in Biot's theory, Sahay (2013) derived an underlying porosity perturbation equation. These three kinematic variables can be changed by a combination of the fluid pressure $\left(p^{\mathrm{f}}\right)$ and the confining pressure $\left(p^{\mathrm{c}}\right)$. The latter is equal to the total pressure

$$
p^{\mathrm{c}}=\eta_{0} p^{\mathrm{f}}+\left(1-\eta_{0}\right) p^{\mathrm{s}}
$$


Herein $\left(1-\eta_{0}\right) p^{\mathrm{s}}$ represents the macroscopic pressure of the solid phase which is related to the macroscopic solid stress tensor $\tau_{j k}^{\mathrm{s}}$ appearing in Biot's constitutive equations (Biot and Willis, 1957) via $-\left(1-\eta_{0}\right) p^{\mathrm{s}}=1 / 3 \tau_{k k}^{\mathrm{s}}$.

For macroscopically homogeneous porous material and in the realm of equilibrium thermodynamics the constitutive poroelastic equations are (Müller and Sahay, 2016a, their equations (16) to (18))

$$
\begin{aligned}
-p^{\mathrm{c}} & =K_{0} \epsilon-\alpha^{\star} p^{\mathrm{f}} \\
-\frac{1}{M^{\star}} p^{\mathrm{f}} & =\alpha \epsilon-\zeta .
\end{aligned}
$$

and the underpinning porosity perturbation equation is

$$
\eta-\eta_{0}=-\frac{\alpha-\eta_{0}}{K_{0}}\left(p^{\mathrm{c}}-\left(\eta_{0}+\left(1-\eta_{0}\right) n\right) p^{\mathrm{f}}\right)
$$

In equation (2) $K_{0}$ is the drained frame bulk modulus and

$$
\frac{1}{M^{\star}}=\frac{\eta_{0}}{K_{\mathrm{f}}}+\frac{\alpha^{\star}-\eta_{0}}{K_{\mathrm{s}}}
$$

is the fluid storage coefficient. The bulk moduli of the solid and fluid phase are $K_{\mathrm{s}}$ and $K_{\mathrm{f}}$, respectively. The porosity perturbation equation entails the parameter $n$ as a macroscopic measure for micro-inhomogeneity and differs from the Biot porosity equation in that the porosity change is not simply determined by the difference pressure (Sahay, 2013). The constitutive equations (2) and (3) also differ from the Biot constitutive equations in that the Biot coefficient $(\alpha)$ and the effective pressure coefficient for the bulk volume $\left(\alpha^{\star}\right)$ are taken as distinct quantities (Müller and Sahay, 2016b) as well as the fluid storage coefficient involves the effective pressure coefficient for the bulk volume. These constitutive equations are based on the volume averaging approach of poroelasticity developed in Sahay et al. (2001); see also Sahay (2013) for further details. 


\subsection{Moduli relevant for drained rock characterization and their micromechanical representation}

Here we focus on drained rock moduli and their interrelations. Drained behaviour refers to a state where the fluid pressure remains constant during the deformation process. From a micromechanical point of view, the bulk volume $\left(V_{\mathrm{b}}\right)$ is the sum of the volume of the solid phase $\left(V_{\mathrm{s}}\right)$ and the pore volume $\left(V_{\mathrm{p}}\right), V_{\mathrm{b}}=V_{\mathrm{s}}+V_{p}$ and the porosity is given by the ratio $\eta=V_{\mathrm{p}} / V_{\mathrm{b}}$. Then, to first order, pore volume changes are composed of bulk volume changes and a change in porosity,

$$
d V_{\mathrm{p}}=\eta_{0} d V_{\mathrm{b}}+V_{\mathrm{b} 0} d \eta
$$

where the subscript 0 refers to the unperturbed (before deformation) value. Thus, in a drained experiment (setting the fluid pressure perturbation to zero) this pore volume change becomes

$$
\left.\frac{\partial V_{\mathrm{p}}}{\partial p^{\mathrm{c}}}\right|_{p^{\mathrm{f}}=0}=\left.\eta_{0} \frac{\partial V_{\mathrm{b}}}{\partial p^{\mathrm{c}}}\right|_{p^{\mathrm{f}=0}}+\left.V_{\mathrm{b} 0} \frac{\partial \eta}{\partial p^{\mathrm{c}}}\right|_{p^{\mathrm{f}}=0}
$$

Multiplying by $1 / V_{\mathrm{p} 0}$ the left-hand side is by definition the drained pore modulus

$$
-\left.\frac{1}{V_{\mathrm{p} 0}} \frac{\partial V_{\mathrm{p}}}{\partial p^{\mathrm{c}}}\right|_{p^{\mathrm{f}}=0} \equiv \frac{1}{K_{\mathrm{p}}} .
$$

It is a measure of how much the pore volume changes in response to a change in confining pressure.

We note that it is sometimes referred to as the pore compressibility $C_{\mathrm{pc}}=1 / K_{\mathrm{p}}$ (e.g. Jaeger et al., 2007).

The first term on the right-hand side in equation (7) represents the drained bulk modulus

$$
-\left.\frac{1}{V_{\mathrm{b} 0}} \frac{\partial V_{\mathrm{b}}}{\partial p^{\mathrm{c}}}\right|_{p^{\mathrm{f}}=0} \equiv \frac{1}{K_{0}} .
$$

and its inverse is the drained rock compressibility $C_{\mathrm{bc}}=1 / K_{0}$. It is routinely measured in pressure cell experiments. The second term on the right-hand side in equation (7) can be evaluated from the porosity perturbation equation (4)

$$
\left.\frac{\partial \eta}{\partial p^{\mathrm{c}}}\right|_{p^{\mathrm{f}}=0}=-\frac{\alpha-\eta_{0}}{K_{0}} .
$$

The Biot coefficient, in turn, is defined through the constitutive equation (3)

$$
\left.\frac{\partial \zeta}{\partial \epsilon}\right|_{p^{\mathrm{f}}=0} \equiv \alpha .
$$


This equation implies that a change of the increment of fluid content due to a change in the bulk volume at zero fluid pressure can only depend on the mechanical properties of the porous frame and on the solid grains that constitute this frame. The bulk modulus associated with the porous frame is the drained frame bulk modulus $K_{0}$. The bulk modulus of the solid-phase $K_{\mathrm{s}}$ could be obtained by applying the same load on the inner and outer surfaces of the porous frame. It is thus determined in hydrostatic compression experiment wherein the change of the solid volume $V_{\mathrm{s}}$ is monitored. Thus

$$
-\left.\frac{1}{V_{\mathrm{s} 0}} \frac{\partial V_{\mathrm{s}}}{\partial p^{\mathrm{f}}}\right|_{p^{\mathrm{f}}=p^{\mathrm{c}}} \equiv \frac{1}{K_{\mathrm{s}}} .
$$

This stipulates that the Biot coefficient is expressed in terms of the drained and solid phase bulk moduli

$$
\alpha=1-\frac{K_{0}}{K_{\mathrm{s}}} .
$$

Substituting the last expression for $\alpha$ into equation (10) and the resulting expression into equation (7) and further noting the definitions of the drained bulk and pore modulus yields to the interrelation

$$
\frac{\eta_{0}}{K_{\mathrm{p}}}=\frac{1}{K_{0}}-\frac{1}{K_{\mathrm{s}}}
$$

Equations (13) and (14) allow us to determine the Biot coefficient and the drained pore modulus for a given porosity, drained bulk modulus and the solid phase bulk modulus. We note that equation (14) is difficult to verify in laboratory experiments because the pore and solid phase bulk modulus would require to measure the changes in pore and solid volume (while typically the bulk volume changes are monitored only).

From a micromechanical point of view the increment of fluid content is (Wang, 2000, equation 2.10)

$$
\zeta=\frac{1}{V_{\mathrm{b}_{0}}}\left(d V_{\mathrm{p}}-d V_{\mathrm{f}}\right)
$$

It can be interpreted as follows. If the pore volume is increased during deformation and is larger than the fluid volume increase then additional fluid can be accommodated in the porous medium. Or, if the fluid volume is reduced by compression and the pore volume remains constant then also more fluid will be stored in the porous medium. Thus it is the difference in pore and fluid 
volume changes that quantifies the change of fluid content of a porous medium. This means that $\zeta>0$ signifies fluid is imported and $\zeta<0$ signifies fluid is expelled from the porous medium. In drained condition the fluid volume cannot be compressed, as this would result in a change in fluid pressure whereas, by definition, drained deformation implies a constant fluid pressure (i. e. zero fluid pressure perturbation). Hence, $\zeta^{\text {drained }}=1 /\left.V_{\mathrm{b} 0} d V_{\mathrm{p}}\right|_{p^{\mathrm{f}}=0}$. Therefore, based on the definitions of the Biot coefficient (11), the drained pore and bulk modulus, Eqs. (9) and (8), we obtain

$$
\alpha=\left.\frac{d V_{\mathrm{p}}}{d V_{\mathrm{b}}}\right|_{p^{\mathrm{f}}=0}=\eta_{0} \frac{K_{0}}{K_{\mathrm{p}}} .
$$

Formally, this equation also follows from Eq. (14). However, to arrive at equation (16) the notion of the solid phase bulk modulus is immaterial.

In the following we estimate the drained pore modulus $K_{\mathrm{p}}$ and the Biot coefficient based on pore-scale simulations in two different ways: First, indirectly through equations (14) and (13). This requires to numerically determine porosity $\eta_{0}$, drained bulk modulus $K_{0}$ and solid phase bulk modulus $K_{\mathrm{s}}$. Second, directly by computing the pore volume changes and using the expressions (8) and (16). We then compare and discuss our estimated values of $K_{\mathrm{p}}$ and $\alpha$ obtained from the two independent analyses.

\section{DIGITAL ROCK SIMULATIONS AND PORE VOLUME CHANGE ANALYSIS}

\subsection{General workflow description and properties of the network of grains}

Typically, there are two types of workflows in DRT. In the first one, micro-structural information is obtained by analysing micro CT scan images with the help of image processing methods. The second workflow consists of multi-physics modelling where 2D micro CT scan images are stacked to create a 3D digital sample replicating the real micro-structure. We implement the second workflow. This includes segmentation of the images into separable pore and grain spaces. Pore spaces are used to calculate the petro-physical properties like permeability, porosity, relative permeability and residual fluid distribution within the rock sample. The framework of grains is subjected to stress and strain and effective elastic properties like grain, bulk and pore modulus are calculated. 
This paper describes the estimation of drained bulk and pore modulus and Biot coefficient of a real rock sample using two different methods. It is an extension of the work of Ahmed et al. (2017).

The numerical workflow includes a digitized rock sample (Ottawa F-42 sand pack) obtained from X-ray micro-computer tomographic (CT) images. 2D micro-CT image slices are stacked together to generate 3D F-42 sand pack samples of different sizeswith a voxel size of $5.78 \mu \mathrm{m}$. We aim to use a real yet idealized and simple rock sample to determine the macroscopic deformation parameters.

The governing equation for the domain of the solid phase (network of grains) is the linear momentum conservation equation. It is solved on structural elements using the commercial software package ABAQUS. The grain material is assumed to be linear elastic, isotropic and homogeneous. It has a density of $2650 \mathrm{~kg} / \mathrm{m}^{3}$, a Young's modulus and Poisson's ratio of $E_{\mathrm{s}}=94 \mathrm{GPa}$ and $\nu_{\mathrm{s}}=0.075$, respectively. This resembles a solid frame made out of quartz with bulk and shear modulus $K_{\mathrm{s}}=36.83 \mathrm{GPa}$ and $\mu_{\mathrm{s}}=44 \mathrm{GPa}$ (Mavko et al. 2008).

\subsection{Representative elementary volume for porosity}

To make the estimates of the macroscopic deformation parameters meaningful, the network of grains must be at least of the size of a representative elementary volume (REV). The REV is estimated by determining the porosity for $100^{3}, 150^{3}, 200^{3}$ and $300^{3}$ voxels. The porosity is calculated using the image processing software AVIZO. The porosity of all samples is found close to 0.33 as shown in Figure (2). The relatively small porosity variations are a consequence of the regularly organized pore structures in the F-42 sand pack. In the following we will use

$$
\eta_{0}=0.33
$$

We are aware of the fact that different porous medium parameters have a different REV, however, we have made no attempt to determine the REV for other quantities than porosity.

\subsection{Solid phase bulk modulus and homogeneous deformation}

Next we calculate the bulk modulus of the solid phase, $K_{\mathrm{s}}$, for all four samples. This is achieved by applying a uniform, positive traction to all faces of the framework of grains, i.e. to all external 
and internal surface elements thereby simulating an all-sided pressure perturbation as implied by its definition (12); see also Figure (3). The pressure is then varied from 1 to $2 \mathrm{MPa}$ and the corresponding volumetric strain is computed. Small pressure values are chosen to make sure that the numerical experiments indeed simulate linear elastic deformation. Figure (4) shows the volumetric strain with pressure for the model with $150^{3}$ voxels. The value determined from the slope is

$$
K_{\mathrm{s}}^{(\mathrm{Eq} \cdot 12)}=36.83 \mathrm{GPa}
$$

This exactly matches the input value of $K_{\mathrm{s}}$ (based on Young's modulus $E_{\mathrm{s}}=94 \mathrm{GPa}$ and Poisson's ratio $\left.\nu_{\mathrm{s}}=0.075\right)$. The same value $\left(K_{\mathrm{s}}=36.83 \mathrm{GPa}\right)$ is obtained for all four sample sizes. While this is the expected result, it serves as a benchmark and validation of the numerical procedure and applied boundary conditions.

We note that the distribution of the volumetric strain is homogeneous throughout the network of grains (Figure 5). This means that this numerical deformation experiment yields to a solid phase bulk modulus that can be considered as a homogeneous deformation modulus.

\subsection{Local volume change analysis}

As ABAQUS uses a mesh grid to discretize the solid phase, in each loading step the Cartesian coordinates of each node as well as the displacement of each node of the mesh, are known. We use these coordinates to estimate the relative variations of the pore and bulk volume. For example, as shown in the Figure 6, each element (here the element is of tetrahedral shape) is changing due to the displacement at all four nodes. Then we calculate the volume of every single element and its changes with respect to the previous loading step. As the element has tetrahedron shape with three polyhedron edge vectors $\mathbf{a}, \mathbf{b}$ and $\mathbf{c}$ the volume of this element $\left(v_{s}\right)$ is

$$
v_{s}=\frac{1}{6}|\mathbf{a} \bullet(\mathbf{b} \times \mathbf{c})| .
$$

Figure (7) shows the volume variations for a sub-volume of the REV under consideration.

To identify and mask those elements which constitute the interior surface (the pore interface; needed to compute the drained pore modulus) and the bounding surface of the REV (needed to compute the drained bulk modulus), we have to define a marker. To do so, we post-process the 
ABAQUS output data and define a geometrical criterion to delineate pore interface and bounding surface elements from intra-grain elements. We use the total number of nearest neighbour elements of each component (i.e. coordination number of the element) to extract pore-interface nodes as well as elements. These neighbourhood numbers are minimum for elements constituting smooth pore interfaces while they are maximum for elements that are fully surround by other elements as is the case in the interior of the solid phase.

\subsection{Drained bulk modulus and inhomogeneous deformation}

Using further simulations we determine the drained bulk modulus $K_{0}$ for all four samples sizes $\left(100^{3}, 150^{3}, 200^{3}\right.$ and $300^{3}$ voxels). This is done by applying a constant traction at the external surfaces but zero traction at the internal grain-pore interfaces thereby simulating an exterior pressure perturbation (Figure 3). We use the same pressure range 1-2 MPa as before. However, due to excessive deformation of some surface elements the numerical solution diverges and hence the simulation cannot be completed. This is understandable, as in this case there is no pressure acting from the inside of the grains so that some of the loose/weakly-connected grains at the surfaces deform heavily and exceed the maximum value that is tolerable in the small deformation limit.

To resolve this issue we change the boundary conditions. We fix one of the six outer surfaces where the large deformation occurs. This make the simulation stable and thus feasible (Figure 8). However, in doing so, we introduce some errors. We simulate our modified value of $K_{\mathrm{s}}$ using the new boundary conditions (one side fixed). The calculated $K_{\mathrm{s}}$ value $(38.4 \mathrm{GPa})$ slightly differed from the previously obtained $K_{\mathrm{s}}$ value $(36.83 \mathrm{GPa})$. The error is $4 \%$ which we consider as acceptable. Therefore, we use this modified boundary conditions in the following. We further restrict the modelling domain to $100^{3}$ voxels.

With this modified boundary condition the sum of the bounding surface elements results in the linear relation shown in Figure (9). Its slope yields to $K_{0}$. However, we have to correct for the missing contribution from the bounding surface that is fixed. This is done by introducing a correction factor $6 / 5$ accounting for the contribution of the sixth outer surface. We obtain

$$
K_{0}^{(\mathrm{Eq} \cdot 9)}=23.4 \mathrm{GPa}
$$


Figure (10a) shows the stress (von Mises stress in MPa) and (10b) volumetric strain distribution during compression of the outer surfaces. In both cases the stress and volumetric strain field is not homogeneous. Both stress and volumetric strain concentration are highest in the vicinity of grain contacts. However, the large volumetric strain values we observe in Figure (10b) are mostly within the solid phase and will not contribute much to the pore-interface strain.

\subsection{Indirect calculation of drained pore modulus and Biot coefficient}

To indirectly estimate $K_{\mathrm{p}}$ we make use of equation (14). This means that we first calculate the solid phase bulk modulus according to its definition (equation 12), for which a uniform, positive traction is applied to all faces of the framework of grains, that is to all external and internal surface elements thereby simulating an all-sided pressure perturbation (pink arrows in Figures 8a). Second, we calculate the drained bulk modulus. Finally, substituting these moduli into equation (14) and taking into account that the porosity is $\eta_{0}=0.33$ we obtain the drained pore modulus

$$
K_{\mathrm{p}}^{\text {ind }}=19.77 \mathrm{GPa}
$$

From equation (13) we also obtain the Biot coefficient

$$
\alpha^{\text {ind }}=0.36
$$

\subsection{Direct calculation of drained pore modulus and Biot coefficient}

During deformation the pore volume changes and the drained pore modulus is the corresponding deformation parameter. To directly estimate $K_{\mathrm{p}}$ we use its micromechanical definition (equation 8). This means that we calculate the volume of each element of the mesh during all loading steps. Then $K_{\mathrm{p}}$ is estimated from the slope of variation of confining pressure $\Delta p^{\mathrm{c}}$ to the relative pore

volume variations $\left(-\frac{\Delta V_{\mathrm{p}}}{V_{\mathrm{p} 0}}\right)$ when the fluid pressure is equal to zero. These relative pore volume variations can be estimated from the change of volume of pore space within a REV.

The volume variation of solid phase is equal and opposite to the pore volume variation. Thus, the pore volume change for each loading step is

$$
\Delta V_{\mathrm{p}}=-\Sigma \delta V_{\mathrm{s}}
$$


where the summation extends over all elements and overall previous loading steps. The pore volume at each loading step is $V_{\mathrm{p}}=V_{\mathrm{p} 0}-\Sigma \delta V_{\mathrm{s}}$. Figure 11 shows that the relative pore volume change

$\left(-\frac{\Delta V_{\mathrm{p}}}{V_{\mathrm{p} 0}}\right)$ depends linearly on pressure acting on the exterior surface of the REV (Figure 8b). Following equation (8), we estimate the drained pore modulus from the slope of the pore volume changes with respect to pressure load. The red curve is the best-fit line to the data. This yields to

$$
K_{\mathrm{p}}^{\mathrm{dir}}=19.02 \mathrm{GPa}
$$

Using (24) the Biot coefficient follows then from equation (16)

$$
\alpha^{\operatorname{dir}}=0.41
$$

which is slightly larger than the indirect estimate $\alpha^{\text {ind }}$.

\section{DISCUSSION}

\subsection{Comparison between the direct and indirect determination of $K_{\mathrm{p}}$ and $\alpha$}

The direct and indirect estimates of drained pore modulus and Biot coefficient differ by $0.75 \mathrm{GPa}$ and 0.05 , respectively. These differences are considered to be within the range of numerical errors. There are different sources of error and limitations in our modelling setup. For the determination of $K_{0}$ the bulk volume changes are computed from the displacements of the outer surfaces of the sample. Given that one of this outer surface is fixed in space (to make the ABAQUS simulations feasible) we introduce another error which we we estimate to be on the order of $1.5 \mathrm{GPa}$ (based on the difference for the solid phase bulk modulus from simulations with and without the fixed outer surface).

Our procedure for the direct calculation of $K_{\mathrm{p}}$ is prone to error because here we assume that the local change in pore volume can be approximated by the change of volumetric strain of a tetrahedral element. The change of volume of the pore space is directly calculated from the solid displacement field at the pore interfaces.

Finally, another source of error is related to the size of the representative elementary volume $(\mathrm{REV})$ that is needed to make the moduli predictions meaningful at the scale of a typical rock 
sample used in laboratory investigations. The chosen REV is based on the REV for porosity which is not necessarily identical to the REV of other poroelastic parameters. The chosen subsample size is relatively small ( $100^{3}$ voxels) and we have not verified how much our predictions would change if larger sample sizes were used.

\subsection{Reality check through comparison with laboratory measurements}

It is interesting to note that De Oliviera et al. (2016) measure the pore volume compressibility for a suite of reservoir sandstones samples. For a sandstone of similar porosity (their Botucatu sandstone sample has porosity 0.3$)$ they report a value of $K_{\mathrm{p}}$ that is significantly smaller ( 0.5 GPa) than our numerical estimate (Figure 12). This comparison is however of limited value as their measurements results are based on uniaxial compression tests rather than hydrostatic compression tests. Jalalh (2006) obtains for a friable reservoir sandstone $\left(\eta_{0}=0.3\right)$ the value $K_{\mathrm{p}}=2.3 \mathrm{GPa}$ at high confining pressure (see Figure 9 in Jalalh, 2006). These lower values of $K_{\mathrm{p}}$ found from laboratory measurements could indicate that our digital rock simulation overestimates the drained pore modulus. This is perhaps not unexpected since digital rock simulations tend to overestimate the drained frame modulus and hence the drained pore modulus when applied to images from consolidated rock samples. The reason is that grain contacts typically add some compliance to the rock skeleton and therefore reduce the overall stiffness. However, in the sand pack images these compliant grain contact areas are not present and therefore a higher $K_{\mathrm{p}}$ appears plausible. Indeed, if compared to the drained pore modulus measurements of Pimienta et al. (2017) for a quartz-rich Bentheimer sandstone sample (their experimental data are also shown in Figure 12) we observe an order-of-magnitude agreement for the highest differential pressure (when presumably the effect of compliant grain contacts become less important). We also remark that the Biot coefficient for Bentheimer sandstone is $\approx 0.4$ at high differential pressure (Figure 10c in Pimienta et al., 2017). This value is rather similar to the numerical estimates presented here. 


\subsection{Importance of pore-interface deformation for poroelastic parameter determination}

The effective elastic properties of digital rocks has been studied and modelled in the elastostatic regime (Arns et al. 2002; Knackstedt et al. 2008; Madadi et al., 2009; Shulakova et al., 2013). Recently, Saxena et al. (2017) have studied the propagation of elastic waves in digital rock samples and have compared the results with elastostatic modelling (NISTs finite element). However, none of these modelling approaches simulates the dynamical process of loading nor do they explicitly characterize the pore-interface deformation and pore volume changes. However, as we demonstrate in this paper, the pore-interface deformation is key in obtaining poroelastic, that is macroscopic, deformation parameters. The advantage of the modelling setup developed here is that we replicate the real deformation process at the pore interface. We are not aware that this has done before in digital rock studies. Nonetheless, the modelling setup is limited to the solid phase deformation. Since the fluid phase is not part of the current modelling the behaviour of two interacting continua is not fully represented. Therefore, undrained poroelastic deformation parameters are not obtained but require an extension of the modelling setup.

\section{CONCLUSION}

We estimate the drained pore and bulk modulus and the modulus of the solid phase and the Biot coefficient for digitized images of a sand pack using numerical simulations. We verify the interrelation among the moduli using poroelasticity theory using an idealized porous rock model and find a reasonable agreement between the simulations. Our results give further impetus in the determination of relevant poromechanical parameters using digital rock technology, particularly those that are difficult to assess in the laboratory. The comparison with other laboratory measurements for consolidated sandstones of similar porosity indicate the drained pore modulus is too high. The reason for this discrepancy is probably the absence of compliant grain contact areas in the digitized sand pack images. 


\section{ACKNOWLEDGMENTS}

We appreciate the constructive feedback and encouragement of S. Shapiro and G. Couples during the 6th Biot conference on poromechanics on an earlier version of this work.

\section{REFERENCES}

ABAQUS, https://www.3ds.com/products-services/simulia/products/abaqus/

Ahmed, S., Müller, T. M., Liang, J., Tang, G., and Madadi, M., 2017. Macroscopic deformation moduli of porous rock: Insights from digital image pore-scale simulations. 6th Biot conference on poromechanics, July 9-13, 2017, Paris, France.

Altmann, J., Müller, T. M., Müller, B. I. R., Tingay, M. R. P., and Heidbach, O., 2010. Poroelastic contribution to the reservoir stress path: International Journal of Rock Mechanics and Mining Science, 47, 11041113 ,

Arns, C. H., Knackstedt, M. A., Pinczewski, W. V., and Garboczi, E. J., 2002. Computation of linear elastic properties from microtomographic images: Methodology and agreement between theory and experiment. Geophysics, 67(5), 1396-1405.

AVIZO, https://www.fei.com/software/avizo-for-materials-science/

Biot, M.A., 1941. General theory for three-dimensional consolidation. Journal of Applied Physics, 12, $155-164$.

Biot, M.A., and Willis, D.G., 1957. The elastic coefficients of the theory of consolidation. Journal of Applied Mechanics, 24, 594601.

Cheng, A.H.-D., 2016. Poroelasticity, Springer, New York.

Couples, G. D., 2017, Idealised discrete pore-scale model of poro-elasticity via closed-form analytical expressions. 6th Biot conference on poromechanics, July 9-13, 2017, Paris, France.

De Oliviera, G.P., Ceia, M.A.R., Missagia, R.M., Archilha, N.L., Figueiredo, L., Santos, V.H., and Lima Neto, I., 2016. Pore volume compressibilities of sandstones and carbonates from Helium porosimetry measurements. Journal of Petroleum Science and Engineering, 137, 185-201.

Jaeger J.C., Cook N.G.W. and Zimmerman R.W., 2007. Fundamentals of Rock Mechanics. Blackwell.

Jalalh, A.A., 2006. Compressibility of porous rocks: Part I. Measurements of Hungarian reservoir rock samples. Acta Geophysica, 54, 319-332.

Knackstedt, M. A., Latham, S., Madadi, M., Sheppard, A. and Varslot, T., 2009. Digital rock physics: 3D imaging of core material and correlations to acoustic and properties. The Leading Edge, 260.

Madadi, M., Jones, A. C., Arns, C. H. and Knackstedt, M. A., 2009. 3D imaging and simulation of elastic properties of porous materials. Computing in Science and Engineering, 11(4):6573.

Makhnenko, R.Y., and Labuz, J.F., 2016. Elastic and inelastic deformation of fluid-saturated rock. Phil. Trans. R. Soc. A, 374, 20150422. 
Mavko, G., Dvorkin, J. Mukerji, T., 2008. Rock Physics Handbook: Tools for Analysis of Porous Media, Cambridge.

Müller, T. M., and P. N. Sahay, 2013. Porosity perturbations and poroelastic compressibilities. Geophysics, 78, no. 1, A7-A11.

Müller, T.M., and Sahay, P.N., 2016a. Generalized poroelasticity framework for micro-inhomogeneous rocks. Geophysical Prospecting, 64, 1122-1134.

Müller, T.M., and Sahay, P.N., 2016b. Biot coefficient is distinct from effective pressure coefficient. Geophysics, 81, L27-L33.

Omdal, E., M. Madland, H. Breivik, K. Naess, R. Korsnes, A. Hiorth, and T. Kristiansen, 2009. Experimental investigation of the effective stress coefficient for various high porosity outcrop chalks: Presented at the 43rd US Rock Mechanics Symposium and 4th U.S.-Canada Rock Mechanics Symposium, 14.

Pimienta, L., J. Fortin, and Y. Guéguen, 2017. New method for measuring compressibility and poroelasticity coefficients in porous and permeable rocks. J. Geophys. Res. Solid Earth, 122, 2670-2689,

Sahay, P. N., T. J. T. Spanos, and V. de la Cruz, 2001. Seismic wave propagation in inhomogeneous and anisotropic porous media. Geophysical Journal International, 145, 209-222.

Sahay P.N. 2013. Biot constitutive relation and porosity perturbation equation. Geophysics 78, L57-L67.

Saxena, N., Saenger, E. H., Hofmann, R. and Wiegmann, A., 2017. Influence of stress and strain loading conditions on elastic and sonic properties of digital rocks. SEG Technical Program Expanded Abstracts, 3882-3887.

Schutjens, P. and Heidug, W., 2012. On the pore volume compressibility and its application as a petrophysical parameter. 9th Biennial International Conference \& Exposition on Petroleum Geophysics, P-512, $1-17$.

Shulakova, V., Pervukhina, M., Müller, T.M., Lebedev, M., Mayo, S., Schmid, S., Clennell, B., Gurevich, B., 2013. Computational elastic up-scaling of sandstone on the basis of X-ray micro-tomographic images. Geophysical Prospecting, 61, 287-301

Tan, X., Konietzky, H., 2014. Numerical study of variation in Biot's coefficient with respect to microstructure of rocks. Tectonophysics, 610, 159-171.

Wang, H. F., 2000. Theory of Linear Poroelasticity, Princeton University Press, New Jersey. 


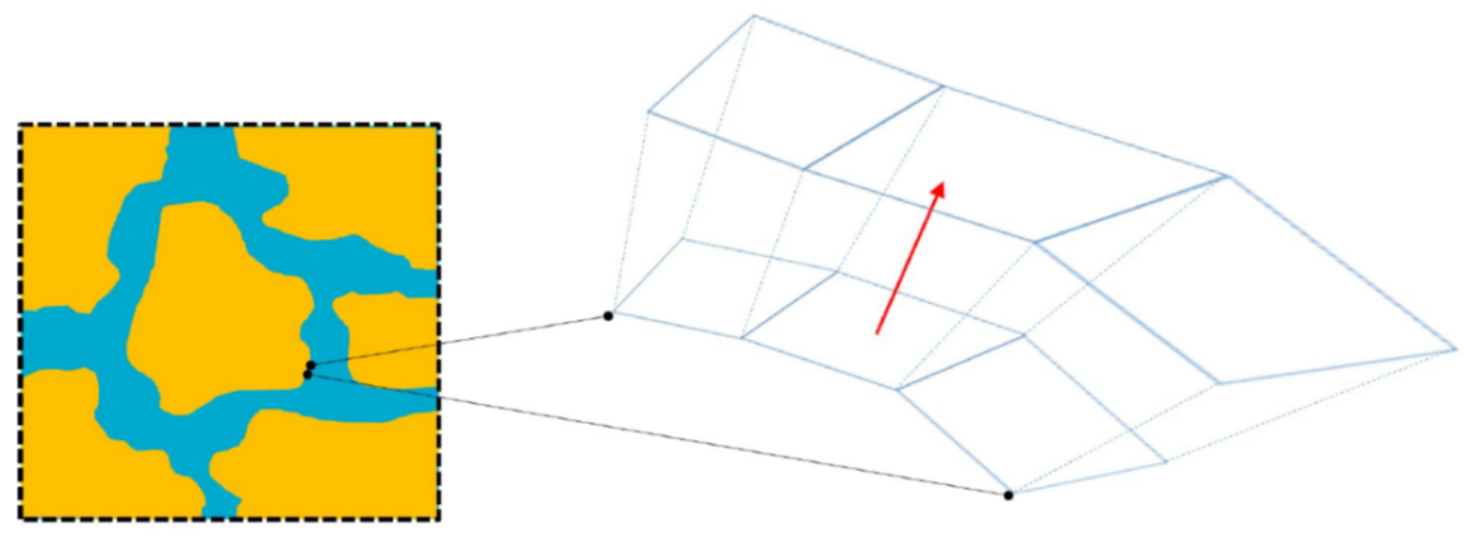

Figure 1. Sketch of a fluid-saturated porous rock at pore scale (left) and oriented surface element with normal of the pore-interface before and after (infinitesimal) deformation (right). At macroscale, there is a porosity change due to the displacement in the direction of the surface normal. 


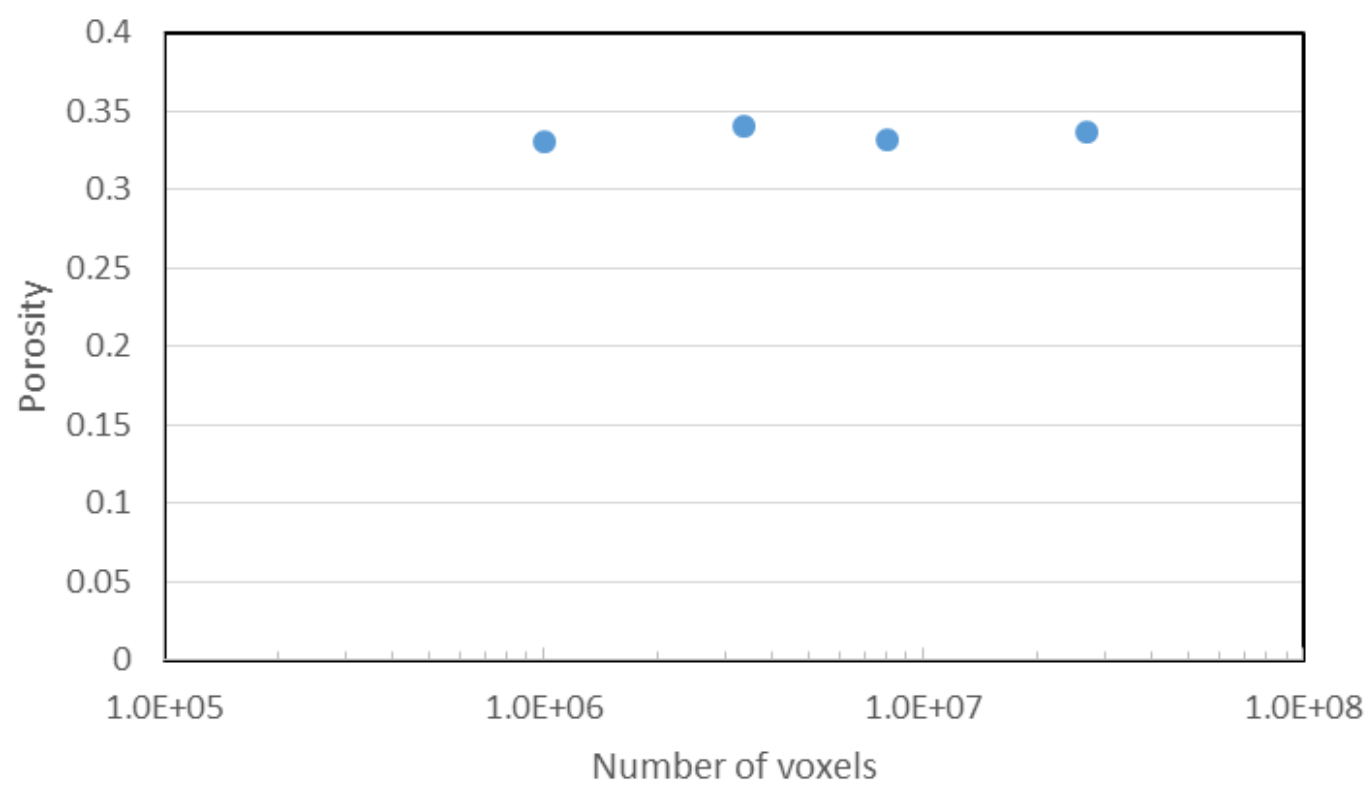

Figure 2. Porosity variation with number of voxels. 


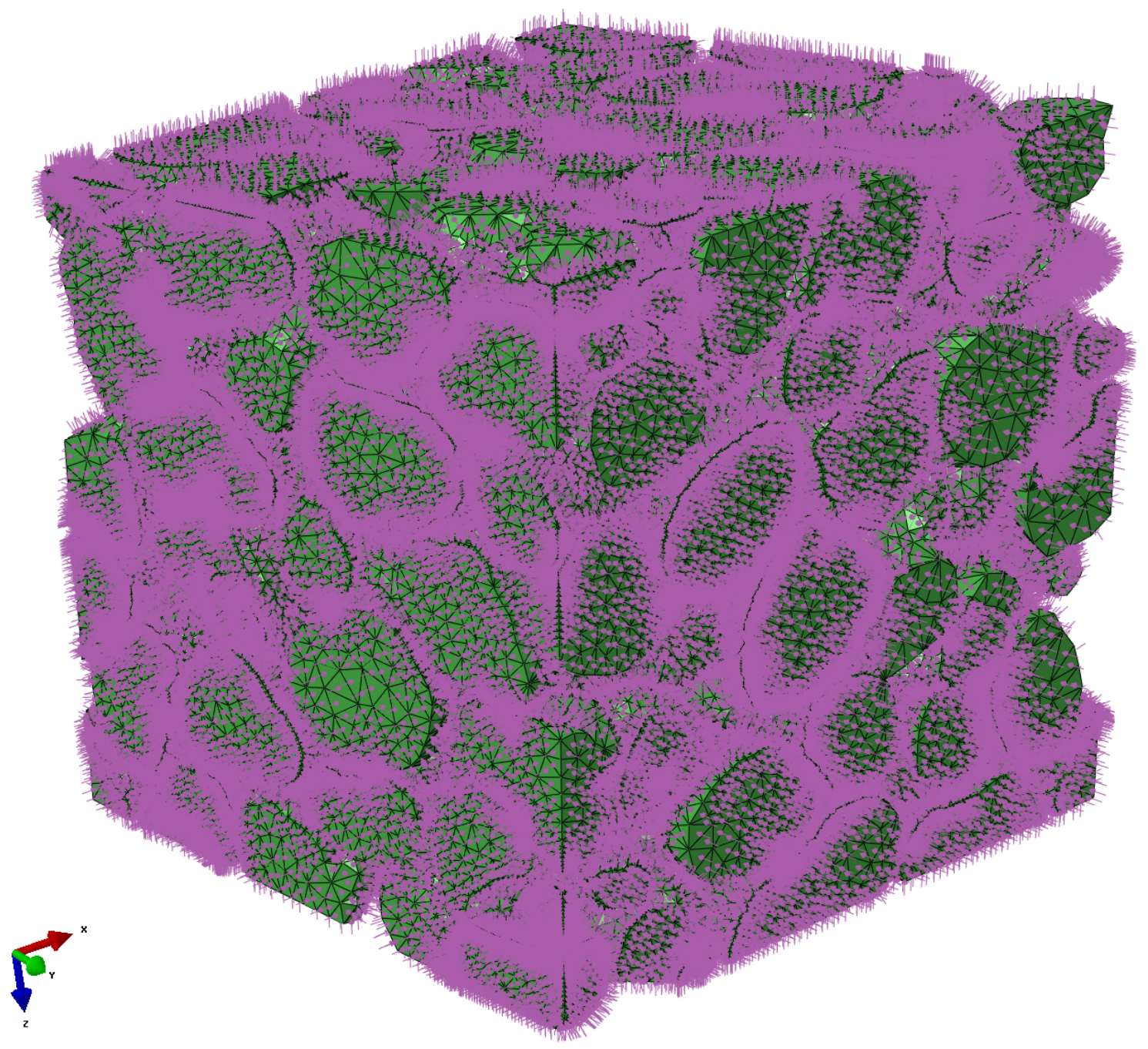

Figure 3. Network of grains constituting the solid phase. Shown are the volume mesh used for grain analysis and the traction vectors (pink arrows) applied to all elements of the outer surface as well as the interior pore surface elements. 


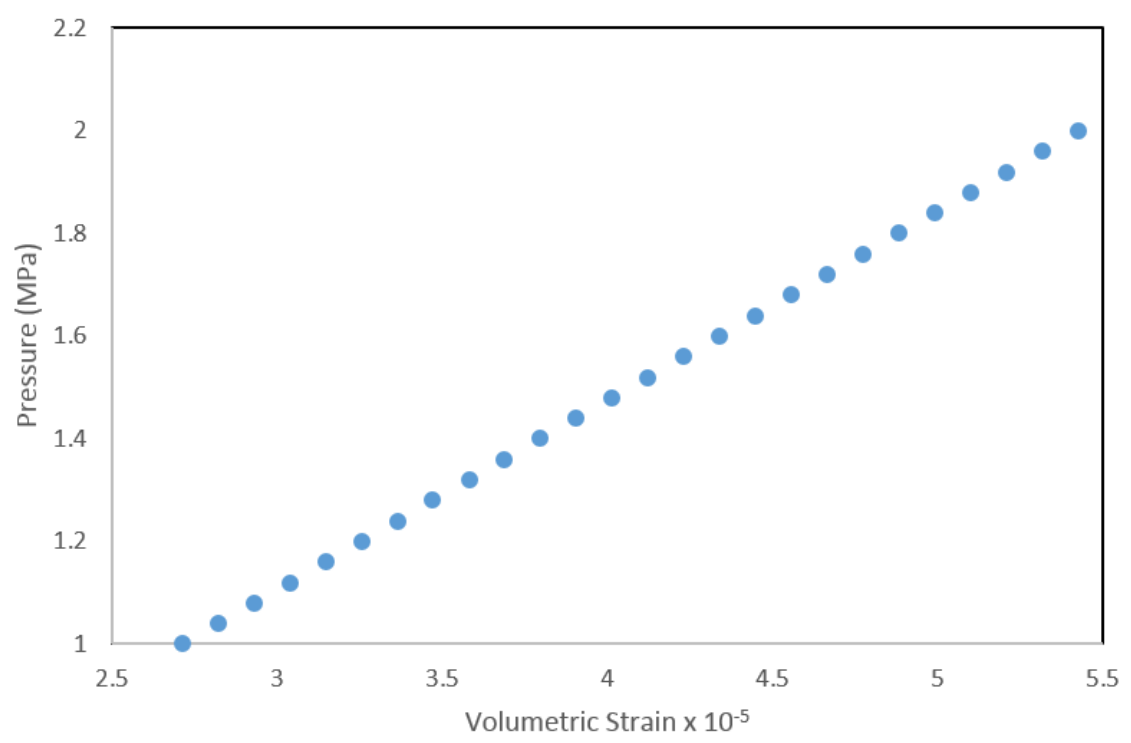

Figure 4. The solid phase modulus is determined from the slope of the pressure perturbation versus volumetric stain curve. Note that this relation is perfectly linear for the applied range of pressures. 

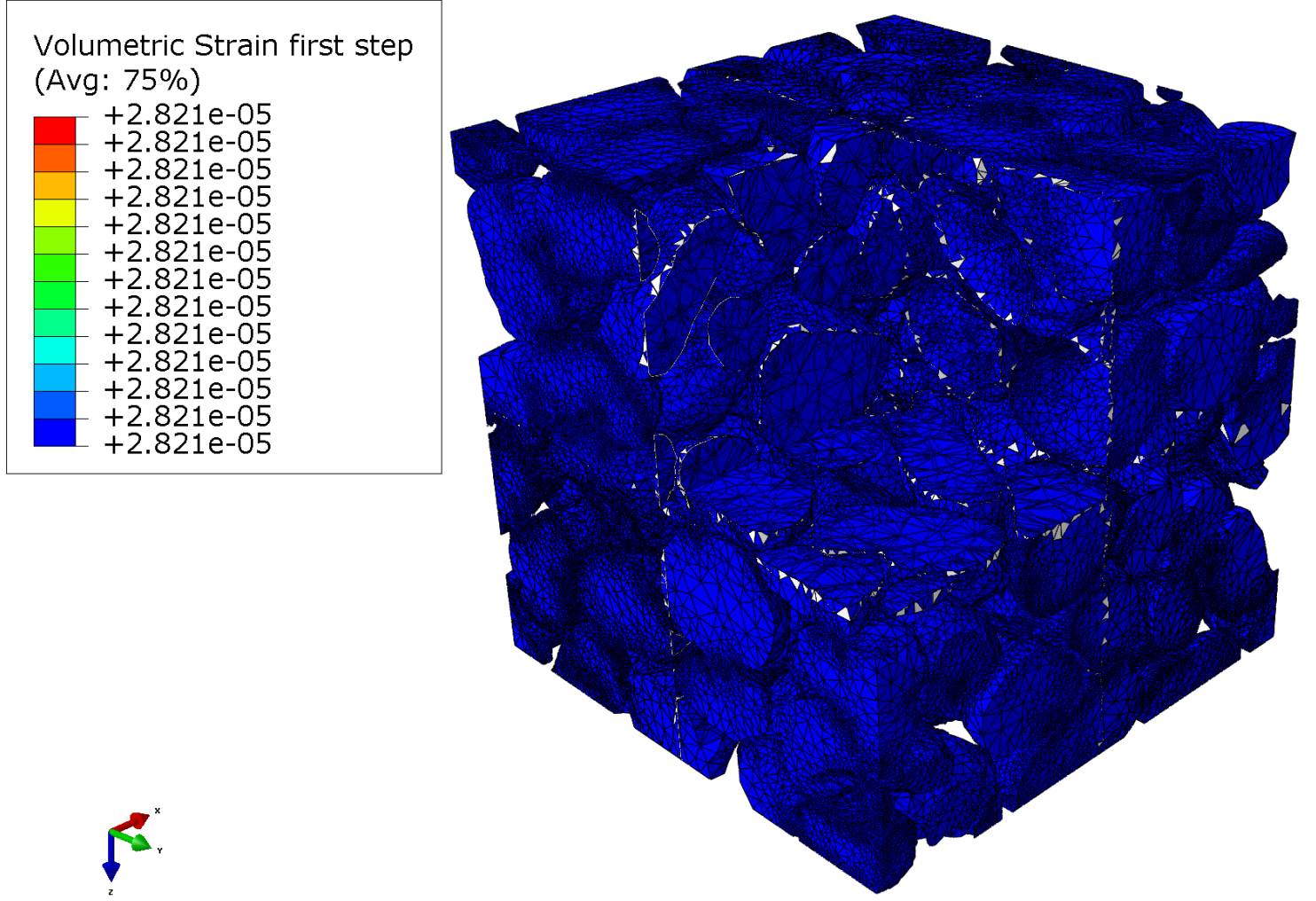

(a)
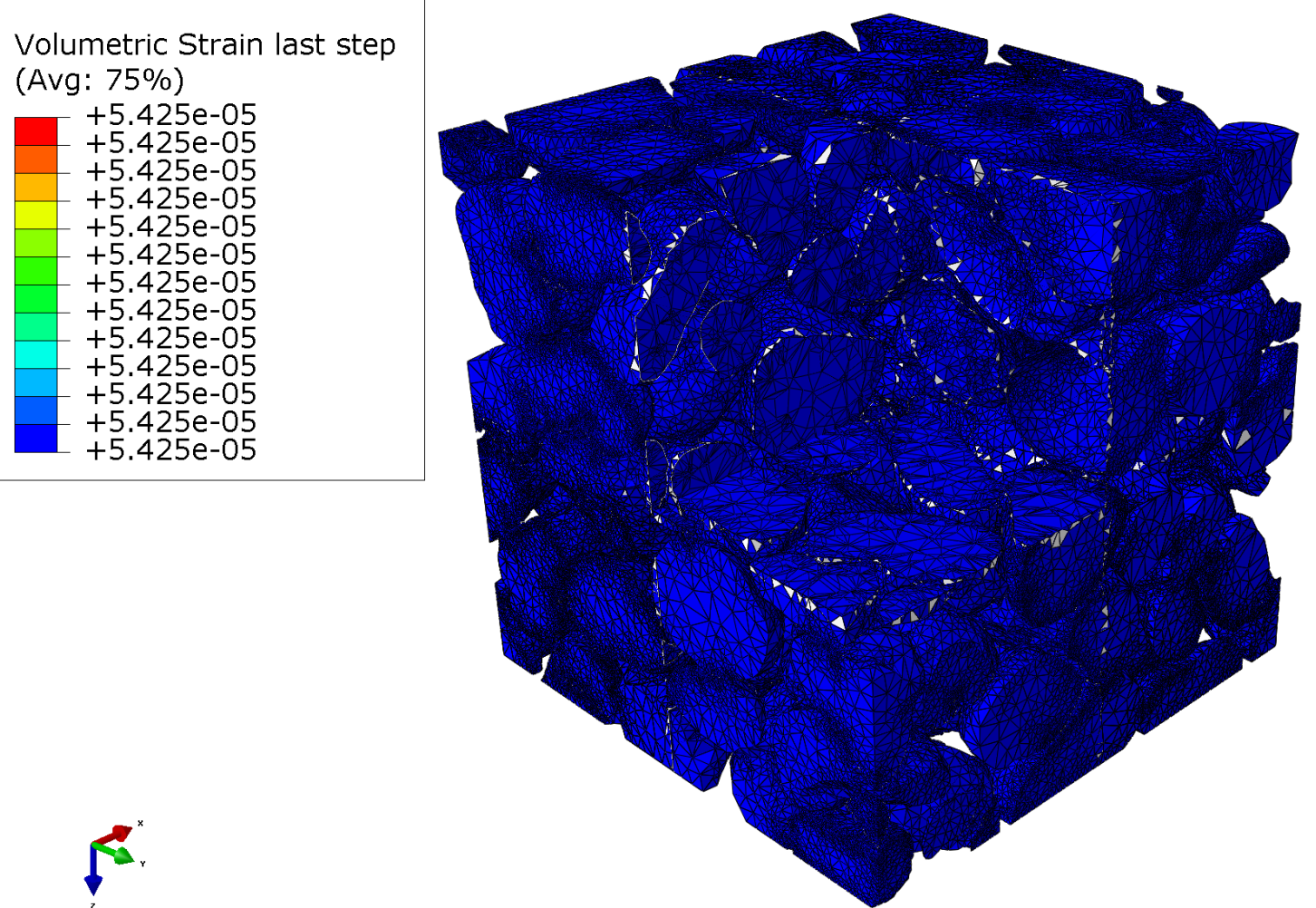

(b)

Figure 5. Volumetric strain distribution during all-sided compression simulation; (a) for the first loading pressure and (b) for the last loading pressure. Note that the pore-scale volumetric strain is homogeneously distributed so that a state of homogeneous deformation is achieved. 


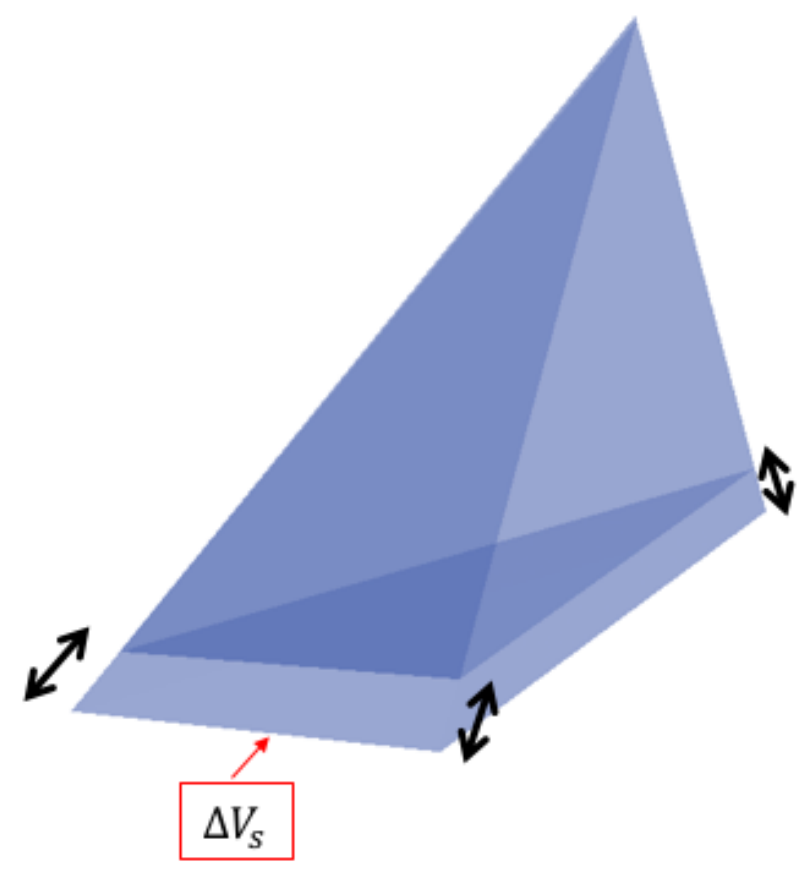

Figure 6. Tetrahedral volume element and its deformation-induced change. 


\section{Ahmed et al.}

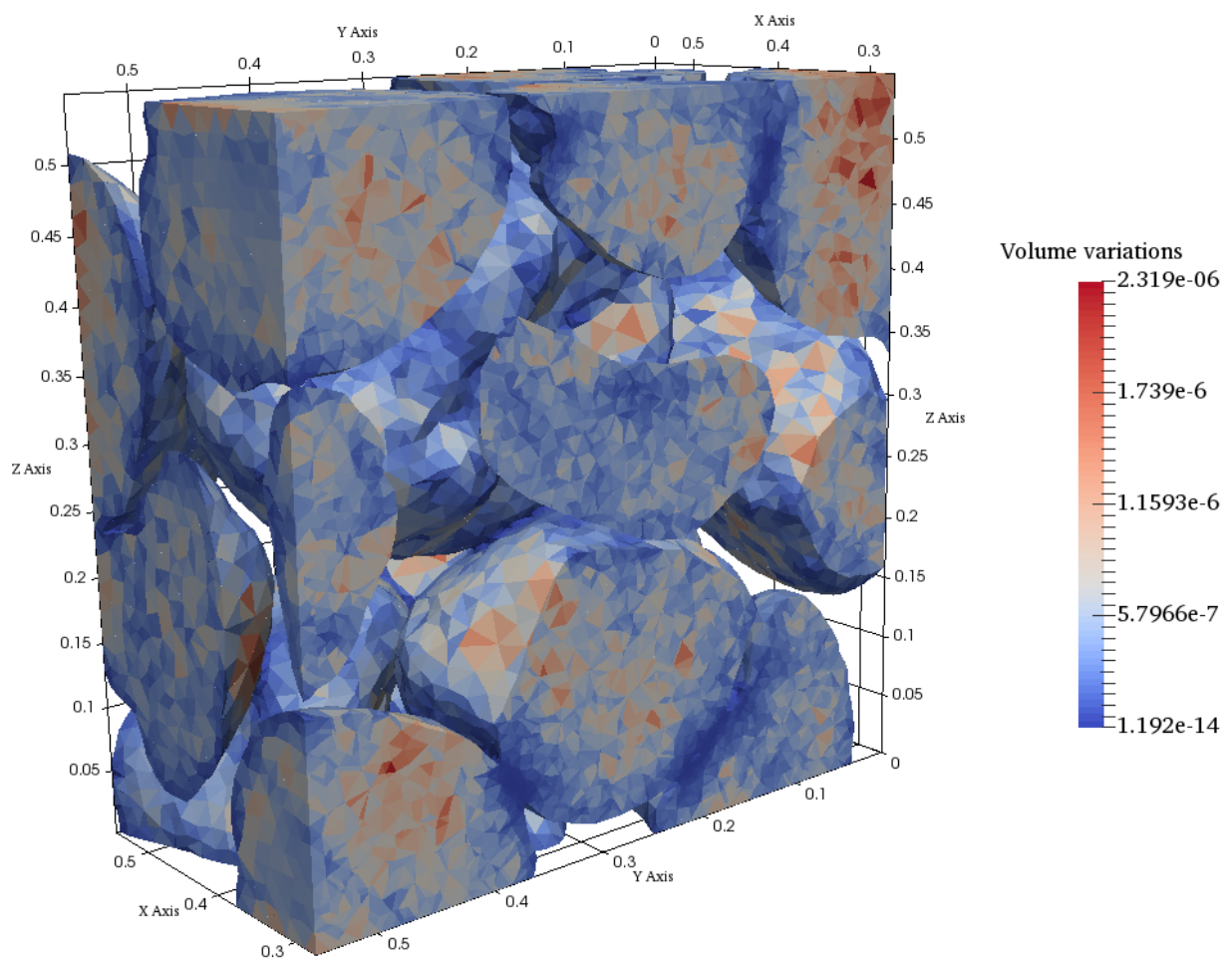

Figure 7. Local volume variations ( $\Delta V_{s}$ in Figure 6) for pore-interface elements and also interior elements for the case when tractions applied to the outer surfaces but no tractions applied to the pore interfaces. 


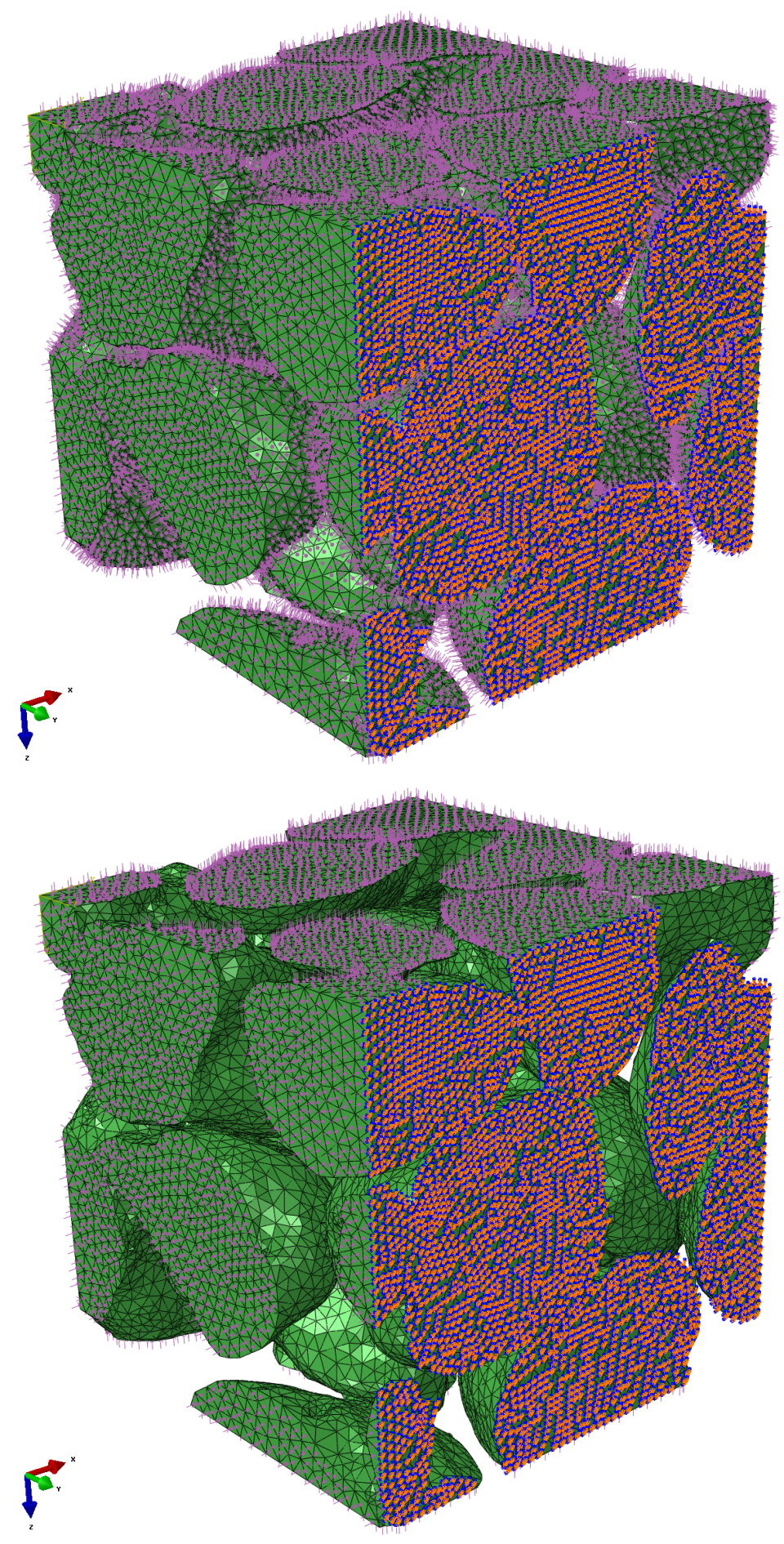

(a)

(b)

Figure 8. Modified boundary conditions with one outer surface fixed in space used for determination of the (a) solid phase bulk modulus via all-sided compression and (b) drained bulk modulus via compression of the remaining five outer surfaces. 


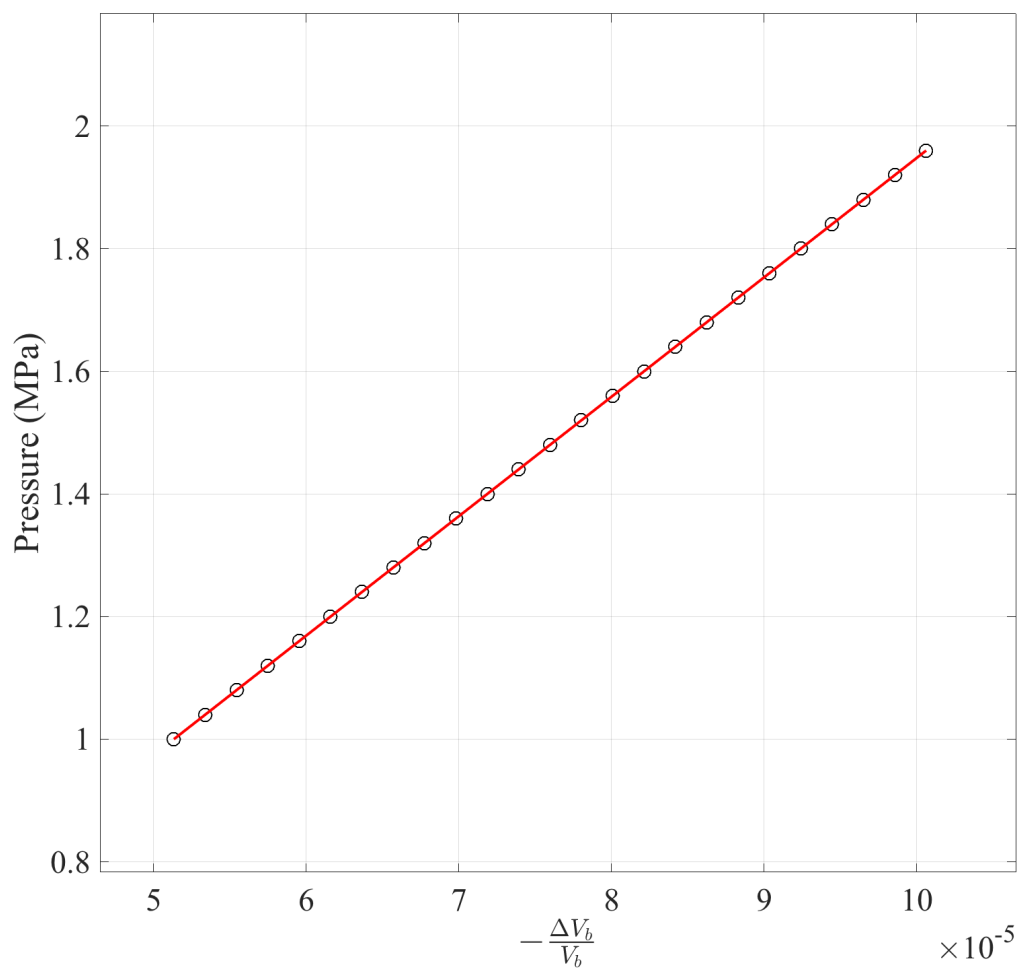

Figure 9. Applied pressure perturbation versus computed volumetric strain. The slope determines the drained bulk modulus $K_{0}$. 


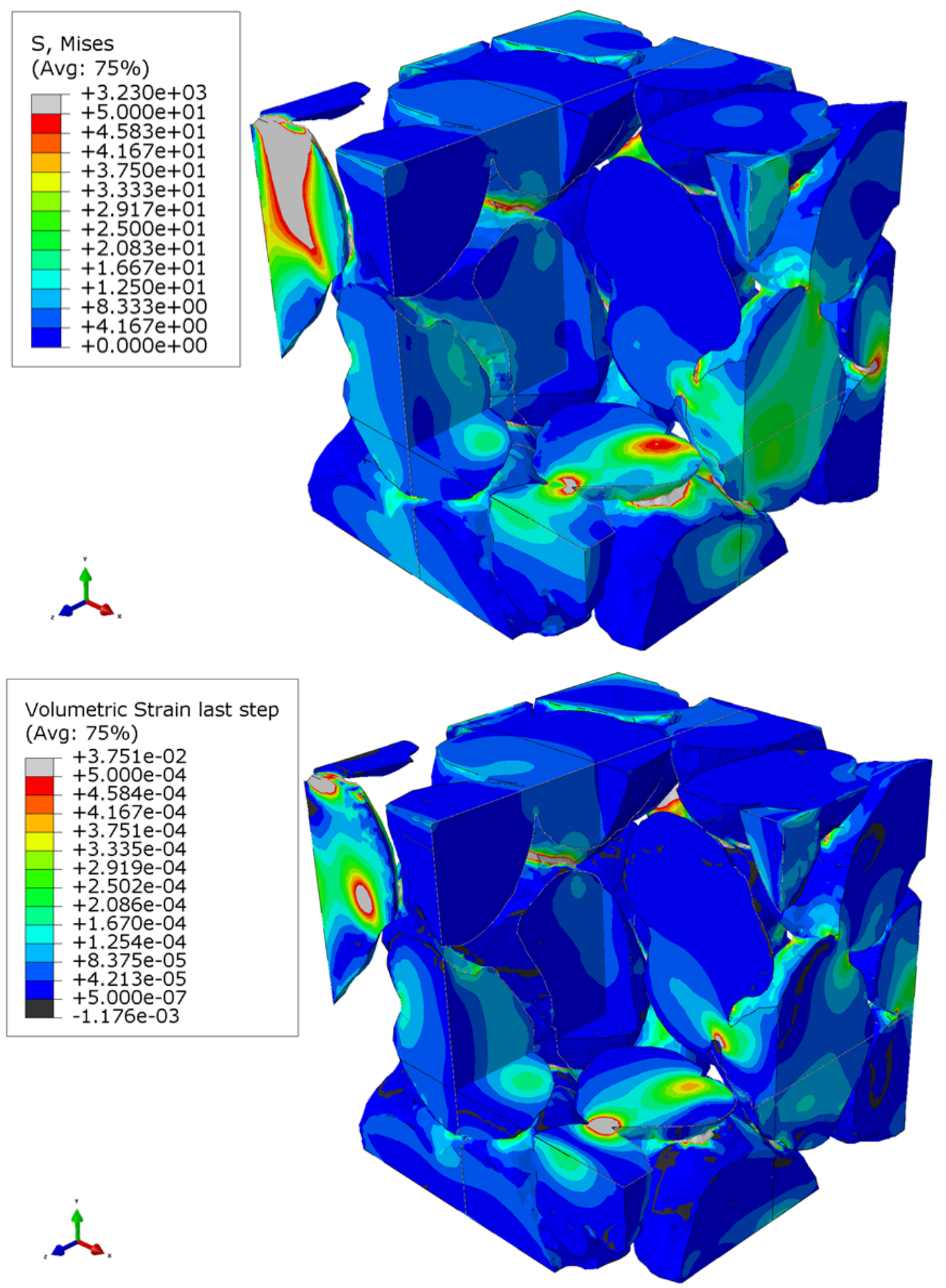

(a)

(b)

Figure 10. Pore-scale (a) stress (von Mises stress $S=\sqrt{3 / 2 \sigma_{i j}^{\mathrm{s}} \sigma_{i j}^{\mathrm{s}}-1 / 2\left(\sigma_{i i}^{\mathrm{s}}\right)^{2}}$ in MPa) and (b) volumetric strain distribution during compression of the outer surfaces. Note that the pore-scale stress and volumetric strain are not homogeneously distributed throughout the network of grains. Both stress and volumetric strain concentration are highest in the vicinity of grain contacts. 


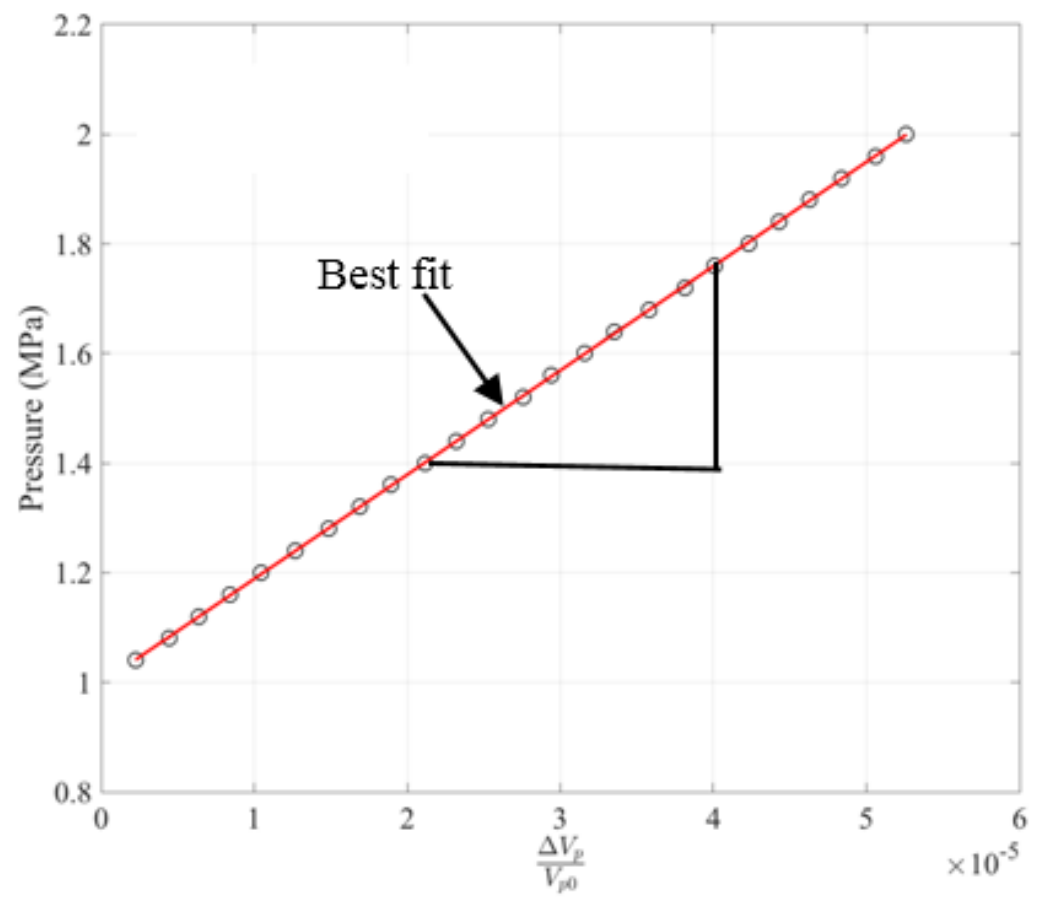

Figure 11. Applied pressure perturbation versus the pore volume changes estimated from the variation of the solid phase volume (Eq. 23) during loading. The drained pore modulus $K_{\mathrm{p}}$ is directly determined from the slope of the best-fit line. 


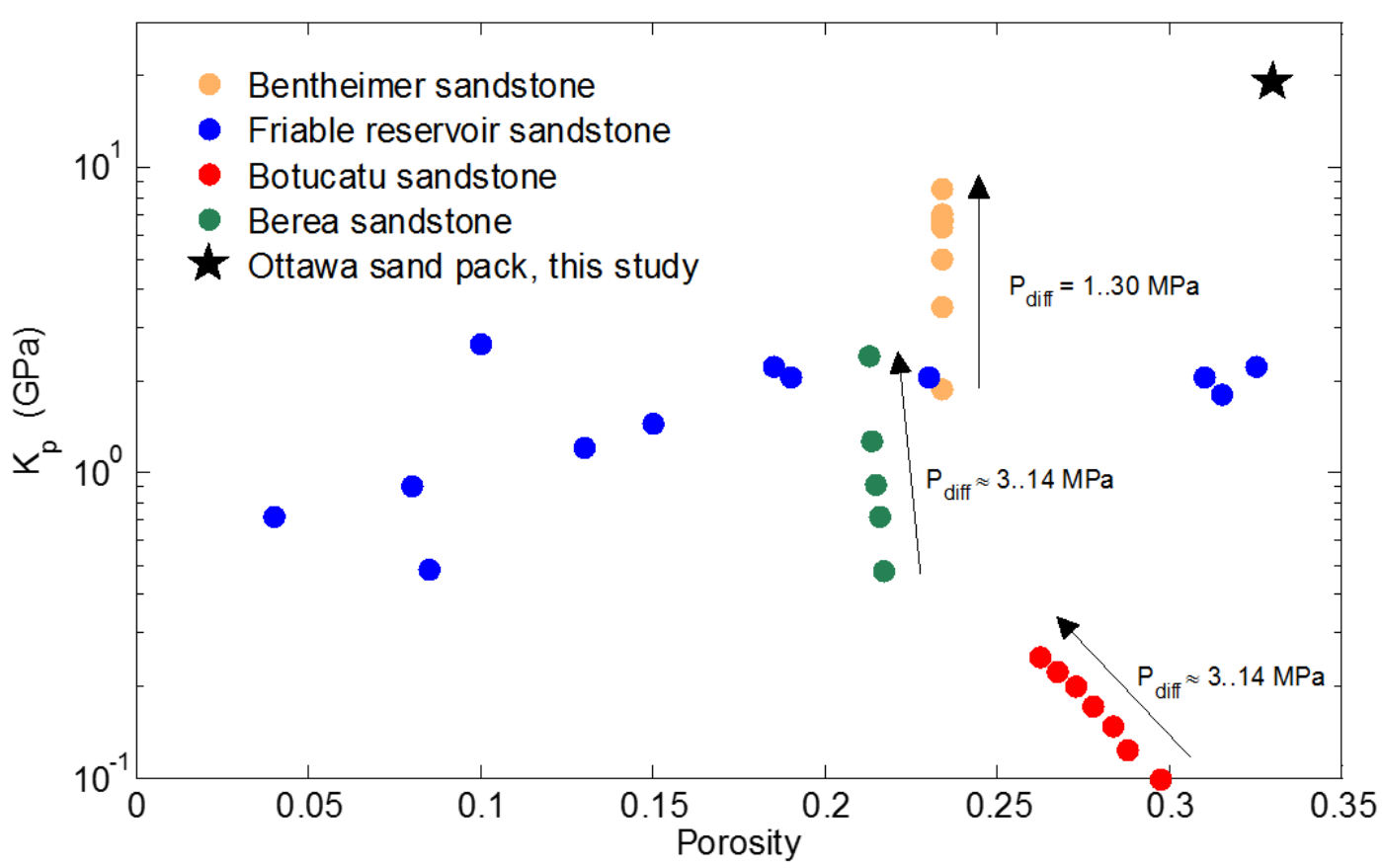

Figure 12. Numerically determined pore modulus compared with laboratory measurements for several sandstones. Blue dots corresponds to samples of variable porosity of a friable reservoir sandstone reported in Jalalh (2006) measured at $\approx 70 M P a$ confining pressure. Red dots represent measurements of a Botucato sandstone sample at varying differential pressure from de Oliviera et al. (2016). As a reference, $K_{\mathrm{p}}$ is shown for a Berea sandstone sample at varying differential pressure (green dots; data taken from de Oliviera et al., 2016). The highest $K_{\mathrm{p}}$ values are reported at high differential pressure for a Bentheimer sandstone sample (Pimienta et al., 2017). 